\title{
Vertrauens-Bildung
}

\author{
Überlegungen zu einer riskanten religionspädagogischen Praxis
}

Thomas Schlag

\section{Zur Beweislage}

Eine aktuelle religionspädagogische Reflexion über die komplexe Bedeutung des Vertrauensbegriffs muss notwendigerweise mit den jüngsten Geschehnissen eines eklatanten, dramatischen und weit reichenden Vertrauensmissbrauchs im pädagogischen Kontext einsetzen.

Symbolhaft kam dieser am 14. Mai 2010 auf dem Ökumenischen Kirchentag in München zum Vorschein. Als sich der Rektor des Berliner Canisius-Kolleg über die Missbrauchsfälle in der katholischen Kirche äussern wollte, stellte sich der Vorsitzende des «Netzwerks Betroffener von sexualisierter Gewalt» vor das Podium und warf den Veranstaltern vehement vor, nicht die Opfer zu Wort kommen zu lassen, sondern «diejenigen, die die Täter geschützt haben». Dem Rektor rief er zu: «Sie haben versagt, treten Sie ab!». Flugblätter mit Bildern der Opfer wurden auf dem Podium verstreut.

Ein konkreter Vertrauensmissbrauch erhielt plötzlich sein sichtbares Antlitz - ganz und gar gegen die geplante Liturgie der Podiumsdiskussion. Das anwesende Publikum war für einen Moment spürbar verunsichert. Eine nahe gehende und durchaus unbequeme Situation. Intensiver ist protestantische Diskurskultur lange nicht herausgefordert worden.

Denn offenkundig standen in dieser Anklage stellvertretend nicht nur Lehrkräfte bedeutender katholischer Internate und reformpädagogischer Schulen am Pranger, sondern auch evangelische Einrichtungen, Waisenhäuser, Schulen und Internate. Wie sich im Laufe der letzten Monate herausstellte, hat auch hier massiver Missbrauch Jugendlicher durch ihre verantwortlichen Erzieherinnen und Erzieher stattgefunden.

Als ehemaliger "Klosterschüler» an den Seminaren Maulbronn und Blaubeuren führen die Presseberichte und die Zeugnisse der Opfer unweigerlich dazu, die eigene Erinnerung an diese Zeit selbst noch auf mögliche Tatbestände abzuklopfen. War man selbst betroffen, ohne dass man es damals selbst sofort bemerkt hätte, hat 
man möglicherweise sexuelle und andere gewalttätige Übergriffe der Vertrauenspersonen verdrängt, wurde man unter dem Schutzmantel des klösterlichen Lebens zu Dingen gezwungen, denen man aus freien Stücken sicherlich niemals zugestimmt hätte? Oder hat man unter Umständen als Jugendlicher übersehen, dass Klassenkameradinnen und -kameraden betroffen waren? Muss also dieses wesentliche und liebgewordene Teilstück der eigenen Biographie umgeschrieben werden? Nebenbei gefragt - wie erinnert der ehemalige Seminarist und heutige Kollege Ingolf Dalferth seine klösterliche Biographie in Schöntal und Urach?

Glücklicherweise fördert bei mir die eigene Erinnerung keine prekären Erlebnisse oder Begebenheiten hervor. Sicherlich erscheinen im Rückblick manche Ereignisse als sonderbar, etwa Einladungen in die Privaträume eines Repetenten, der sich einen Spass daraus machte, uns mit Hochprozentigem «abzufüllen» oder manche Klagen von Mädchen darüber, dass eine männliche Aufsichtspersonen die abendlichen Kontrollgänge eben auch durch ihre Zimmer machte. Unangenehme Gespräche oder gar übergriffige Berührungen durch die verantwortlichen Erwachsenen sind mir aber jedenfalls nicht in Erinnerung, und dies trotz aller Intimität, die ein solch intensives Zusammenleben auf engem Raum eben mit sich bringt. Vielleicht habe ich einfach Glück gehabt mit dieser evangelischen Einrichtung oder hat sich hier möglicherweise tatsächlich der pädagogische und evangelische Geist gezeigt, der eine solche Einrichtung eben ausmachen sollte und der Sache nach einzig wahr zu sein beanspruchen darf?

Wie auch immer: der Grat zwischen empathischer Annahme und phantasievoller Übergriffigkeit ist dort wohl besonders schmal, wo es eben um die ganze Person geht, insbesondere dann, wenn Erwachsene es eben tagtäglich mit Jugendlichen zu tun haben, die sich im hochemotionalen Suchprozess zwischen Abhängigkeit und Autonomie befinden. Umso fataler ist es, wenn offenbar in Schulen und Einrichtungen, die sich das Ideal vertrauensvoller Persönlichkeitsbildung auf die Fahnen geschrieben haben, seelische und körperliche Vergewaltigungen nicht selten systematisch an der Tagesordnung waren und offenbar sogar mit religiöser Inbrunst legitimiert wurden. Offenbar paarten sich bei diesen massiven Grenzüberschreitungen nicht selten krude und unausgelebte sexuelle Vorstellungen mit bestimmten pädagogischen und religiös verbrämten Allmachtsphantasien.

Insofern erhebt sich über alle anfangs aufgezeigte persönliche Dramatik hinaus und neben allen juristischen und ethischen Fragen 
eines solchen Missbrauchs im pädagogischen Vertrauensraum die Grundfrage, wie sich ein personengemässer, professioneller Umgang mit Nähe und Distanz so fassen lässt, dass einerseits Raum für einen spezifisch evangelischen Bildungsauftrag entsteht, zugleich aber konkrete Gefährdungen auch nur eines einzigen Jugendlichen grundsätzlich ausgeschlossen werden können.

\section{Ein Schritt zurück, um wieder einen Schritt nach vorne gehen zu können}

Aus guten Gründen und zugleich auch pädagogisch wohlbegründet hat die evangelische Religionspädagogik in den vergangenen Jahrzehnten mit ihrem Fokus auf die Subjektorientierung einen wesentlichen Erkenntnis- und Praxissprung geleistet. Nach einer unheilvollen und langen Geschichte katechetischer Unterweisung, in deren Rahmen Kinder und Jugendliche vornehmlich als Objekte autoritärer Belehrung verstanden wurden, ist seit dem letzten Drittel des 20. Jahrhunderts aus dem Bild des unmündigen Zöglings die Vorstellung des mündigen Dialogpartners erwachsen. Aus theologischen und pädagogischen Gründen, so die grundlegende Einsicht, verbietet es sich, Kinder und Jugendliche als leere Gefässe für die Einflössung vermeintlich eindeutiger Inhalte ansehen oder sie gar primär für eine willenlose Einpassung in die Institution Kirche funktionalisieren $\mathrm{zu}$ wollen.

Gegenwärtig zeigt sich dies in umfangreichen Forschungsbereichen unter den Stichworten der Kinder- und Jugendtheologie. Dieses konstitutive Ernstnehmen des lernenden Subjekts im Kontext religionspädagogischer Theoriebildung hat fraglos $\mathrm{zu}$ erheblichen Wandlungsprozessen in der konkreten Unterrichtspraxis geführt, hinter die in keinem Fall wieder zurückzukehren ist. Der Gewinn dieser grundlegenden Blickveränderung bestand und besteht darin, dass in der Tat alle Diskurse über inhaltliche Aspekte der biblischen Überlieferung und auch deren theologische und alltagsbezogene Interpretation keinesfalls unter Absehung jugendlicher Verstehensund Kommunikationsvoraussetzungen erfolgen können.

Was man sich nun aber mit dieser Subjektorientierung als Problem einhandelte, war ein Vorstellung gelingender Bildung, die eben von möglichst persönlichkeitsbezogenen Lern- und Kommunikationsprozessen ausging. Dies verband und verbindet sich mit der Vorstellung der Lehrenden als möglichst vertrauensvoller und vorbildhafter Personen, die durch ihre persönliche Empathie 
und Nähe gleichsam die Plausibilität der einzelnen, oftmals als sperrig empfundenen Lehrstoffe transportieren sollten. Die Bedeutsamkeit einzelner Themen soll also nicht zuletzt durch die persönliche, vertrauensoffene Zugewandtheit der einzelnen Lehrperson mit signalisiert und vergegenständlicht werden.

Interessanterweise finden sich jedoch keine systematischen Abhandlungen zur Vertrauensthematik innerhalb der religionspädagogischen Fachliteratur der letzten Jahrzehnte und auch die einschlägigen Lexika verzeichnen zum Vertrauensbegriff keine Einträge. Die jüngste Orientierungshilfe der EKD spricht eher nur am Rande von einem "gewisse[n] Vertrauensvorschuss für kirchliche Träger besonders dort ..., wo es etwa um Fragen der Werteorientierung geht» und nennt explizit lediglich die "Vertrauenswürdigkeit der Kirche»". In einer der wesentlichen aktuellen Abhandlungen zur Frage der Kompetenzorientierung ist lediglich an einer einzigen Stelle das semantische Feld bespielt und dies ausgerechnet dort, wo von einer "vertrauensförderliche[n] Sitzordnung»" die Rede ist! Und selbst in der jüngsten Handreichung zum Verhältnis von Kirche und Jugend, wo es sich der Sache nach besonders nahe legen würde, taucht der Begriff abgesehen von einem Hinweis auf die Bedeutung «vertrauensvolle[r] Freundschaften $»^{3}$ für Jugendliche - explizit nicht auf.

Es scheint also, als ob derVertrauensaspekt in didaktischer Hinsicht zwar gleichsam implizit immer mit vorausgesetzt ist, aber bisher eine grundlegende Auseinandersetzung in dieser Sache vermieden wird. Angesichts der Tiefgründigkeit des Vertrauensthemas stellt eine systematische Beschäftigung somit ein erhebliches Desiderat für die religionspädagogische Selbstverständigung dar.

Aus meiner Sicht ist deshalb nochmals grundsätzlich neu zu überlegen, wie viel persönliche Nähe und Intimität eine gelingende religiöse Vertrauens-Bildung bedarf. Denn ohne Frage ist die Zielsetzung einer Bildung, die auf Vertrauen setzt und selbiges zu erzeugen sucht, ein in hohem Mass riskantes Unterfangen. Denn das Angebot einer «nahe gehenden», auf grösstmögliche Gefühle

\footnotetext{
1 Kirchenamt der EKD (Hg.), Kirche und Bildung. Herausforderungen, Grundsätze und Perspektiven evangelischer Bildungsverantwortung und kirchlichen Bildungshandelns. Eine Orientierungshilfe des Rates der Evangelischen Kirche in Deutschland (EKD), Gütersloh 2009, 57.

2 D. Fischer/V. Elsenbast (Red.), Grundlegende Kompetenzen religiöser Bildung Zur Entwicklung des evangelischen Religionsunterrichts durch Bildungsstandards für den Abschluss der Sekundarstufe I, Münster 2006, 26.

3 Vgl. Kirchenamt der EKD (Hg.), Kirche und Jugend. Lebenslagen - Begegnungsfelder - Perspektiven. Eine Handreichung des Rates der Evangelischen Kirche in Deutschland (EKD), Gütersloh 2010, 24.
} 
abzielenden Pädagogik ist niemals ohne Fallstricke. Damit soll natürlich keinesfalls gesagt werden, dass die anfangs erwähnten Missbrauchsfälle vor dem Horizont subjektorientierter Pädagogik geschahen. Gerade das Gegenteil ist vielmehr der Fall, indem die Jugendlichen eben wieder als bestenfalls unmündige Zöglinge und willenlose Objekte der Begierde angesehen wurden.

Vielmehr sei die These vertreten, dass darüber nachzudenken ist, ob religiöse Bildung als Vertrauens-Bildung möglicherweise nicht per se in der Gefahr steht, persönliche Nähe über Gebühr zum entscheidenden, gar ausschliesslichen Kriterium für gelingenden Unterricht $\mathrm{zu}$ machen, sich damit selbst überhebt und unter der Hand ihre Bildungsklientel emotional und persönlich zu überfordern droht. Notwendig ist es deshalb, das richtigerweise ausgelobte Ziel einer möglichst persönlichkeitsbedeutsamen, vertrauensbasierten religiösen Bildung seinerseits differenziert zu beschreiben und zu verfolgen.

Eine aus meiner Sicht notwendige Differenzierung subjektorientierter Bildung lässt sich am Vertrauens-Begriff in besonders guter Weise religionspädagogisch - und dies heisst theologisch wie pädagogisch - verdeutlichen:

\section{Eine hilfreiche reformatische Grunddifferenzierung}

Für den religionspädagogischen Theorieweg legt es sich nahe, noch einmal neu die reformatorische Grunddifferenzierung von notitia, assensus und fiducia ins Spiel zu bringen. Hilfreich ist diese, da sich durch sie komplexe pädagogische Prozesse in theologischem Sinn ausdifferenzieren lassen. Dies ist nicht zuletzt angesichts einer gewissen PISA-Hysterie zu betonen, in deren Folge in jüngster Zeit primäres Augenmerk auf einen faktenbezogenen Kenntniserwerb gelegt wird. Die weitgreifende Debatte über Bildungsstandards und Kompetenzerwerb betont einerseits zwar wieder zu Recht, dass auch religiöse Bildung auf die Vermittlung basaler Kenntnisse Gewicht zu legen hat, denn in den vergangenen Jahrzehnten ist dies deutlich zu gering betont worden. So ist es sachgemäss, wenn für den Religionsunterricht eine stärkere Betonung von einschlägigem Fachwissen gefordert wird. In der Tat stellt sich dieses schulische Fach vor immense Probleme, wenn Jugendliche ausgerechnet hier den Eindruck gewinnen sollten, als ob es in diesem Fach im Wesentlichen um freie und frei im Raum hängende Diskussionen alltäglicher Lebensprobleme gehen sollte. 
Vergleicht man etwa die inhaltlichen Anforderungen anderer Fächer in der Sekundarstufe, so ist schlechterdings weder zu begründen noch nachzuvollziehen, weshalb nicht auch der Religionsunterricht ein anspruchsvolles und durchaus auch leistungsorientiertes Profil an den Tag legen sollte. Gleiches gilt sicherlich auch für die Konfirmationsarbeit, noch zumal sich die gegenwärtige Generation Jugendlicher durch einen eklatanten Mangel an religiösem Wissen auszeichnet. In diesem Sinn wird durch die durch die PISA-Diskussion ausgelöste Frage nach einer qualitätsvollen religiösen Bildung die wichtige inhaltsbezogene Aufgabe religiöser Bildung zu Recht wieder aufgegriffen und thematisiert. Dies entspricht ohne Frage schon der reformatorischen Forderung, dass ein mündiges Christsein einer breiten Kenntnis wesentlicher Grundlagen von Bibel, Bekenntnis bzw. der notitia als elementarer Kenntnis des Wortes Gottes und seiner theologischen Bedeutung.

Zugleich weist nun aber bereits Luther aus guten theologischen Gründen darauf hin, dass es mit dieser notitia keineswegs getan ist. Vielmehr bedarf es für eine tatsächliche Vertrautheit mit dem Wort Gottes eines eminent persönlichen Zugangs: also des assensus als der persönlichen Zustimmung und des annehmenden Glaubens des Gehörten und Gelesenen im Sinn eines nicht nur kognitiven, sondern eben affektiven, emotionalen und habituellen Einverständnisses mit dem, was in religiösen Bildungs- und Kommunikationsprozessen je als wesentlich aufscheint.

Was nun allerdings als ein Drittes dazu kommen muss, ist die Möglichkeit, diesen Inhalten tatsächlich im Sinn der persönlichen fiducia vertrauen zu können. Oder wie es Melanchthons als definitio fidei umgreift: «Fides est assentiri universo verbo Dei nobis propositio adeoque et promissione gratuitae reconciliationis donatae propter Christum Mediatorem estque fiducia misericordiae Dei promissae propter Christum Mediatorem» ${ }^{4}$.

Gerade von dort her wäre es kurzschlüssig, diese fiducia primär an die erziehende oder lehrende Person zu binden oder gar von deren Glaubwürdigkeit abhängig zu machen.Vielmehr muss der individuelle Erkenntnisprozesse wesentlich auf derVertrauenswürdigkeit der Inhalte selbst basieren, also dem Vertrauen darauf, dass Gott selbst in Wort, Überlieferung und Auslegung durch die Geschichte hindurch treu, barmherzig und verheissungsvoll präsent ist.

Diese reformatorische Grunddifferenzierung bedeutet für die Lehrenden Entlastung und Verantwortung zugleich. Einerseits dürfen und müssen sie den Prozess selbst und sein Gelingen nicht pri-

P. Melanchthon, Loci praecipui theologici (1559), CR 21, 744. 
mär von ihrer eigenen pädagogischen und theologischen Fähigkeit abhängig machen, Vertrauen auszustrahlen oder gar zu erzeugen. Sie sind gerade aufgrund der möglichen Eigen- und Wahrheitsdynamik göttlicher Geschichte vom Anspruch entlastet, für die lebensbedeutsame Plausibilität dessen zu sorgen, wovon ihr Unterricht handelt. Auf der anderen Seite bringt dies für sie die erheblicheVerantwortung mit sich, Bildungsprozesse so zu planen und auszugestalten, dass sie der theologischen Sache selbst möglichst gut durch ihre Handlungsund Kommunikationsweise zu entsprechen vermögen.

\section{Vertrauens-Bildung als beziehungsorientierte Bildung}

Ein guter und qualitätsvoller Unterricht in religiösen und theologischen Fragen sollte also keineswegs auf persönliche Nähe und die Bearbeitung individueller, existentieller Fragen verzichten, sondern im Gegenteil diese als einen wesentlichen Aspekt eines sinnvollen Bildungsgeschehens profilieren. Fatal wäre es, würde man die einzelnen Themen religiöser Bildung primär als einen zu lernenden Unterrichtsstoff verstehen, der womöglich am Ende auch noch überprüfbar abgefragt werden kann.

Allerdings ist dabei die Gefahr von Grenzüberschreitungen immer mit im Auge zu behalten. Sinnvoll für eine permanente Selbstüberprüfung der Lehrenden ist es aus meiner Sicht, Formen vertrauensvoller Bildung ihrerseits entlang des Beziehungsbegriffs zu gestalten. Der Beziehungsbegriff empfiehlt sich aus meiner Sicht gerade deshalb, weil er weit mehr als nur die Beziehung zwischen Lehrendem und Lernendem umfasst. Vielmehr umschliesst dieser in theologischem Sinn eben auch die Beziehung des Lernenden zu sich selbst wie - im Sinn der Melanchthon'schen Differenzierung - die Beziehung Gottes zu den Akteuren innerhalb des konkreten Bildungsgeschehens. Schliesslich ist als eine vierte Beziehungsdimension auch noch die Beziehung des Einzelnen zur Zukunft im Sinn göttlicherVerheissung mitzudenken ${ }^{5}$.

Der Beziehungsbegriff kann also in gutem Sinn dazu dienen, sowohl die notwendige pädagogische Dimension persönlicher Nähe wie auch das über das menschlich Machbare und die aktuell fassbare Zeit hinausgehende Beziehungsgeschehen Gottes mit den Menschen im konkreten Bildungsprozess aufscheinen zu lassen und

5 Vgl. R. Boschki, «Beziehung» als Leitbegriff der Religionspädagogik. Grundlegung einer dialogisch-kreativen Religionsdidaktik, Ostfildern 2003. 
damit die Dialektik von Verfügbarem und Unverfügbarem mitzudenken und mit zu thematisieren. Zugleich gilt aber reformatorisch, dass dieses Unverfügbare eben nicht das schlechthin Kontingente ist, sondern seinerseits von der durch Gottes Wort ausgesprochenen Verheissung her qualifiziert und überwölbt wird.

\section{Vertrauens-Bildung am Beispiel der Gottesfrage}

Die religionspädagogische Kunst menschen- und sachgemässer Vertrauens-Bildung lässt sich anhand der Thematisierung der Gottesfrage exemplarisch konkretisieren. Angesichts der Tendenz, dass religiöse Sozialisationsprozesse im familiären Kontext zunehmend seltener stattfinden oder mindestens weniger selbstverständlich als noch wenige Generationen zuvor ausfallen, besteht die erste Aufgabe religiöser Bildung in der Tat darin, Kinder und Jugendliche mit den vielfältigen und unterschiedlichen Gottesbildern des Alten und Neuen Testaments vertraut zu machen. Narrative und symbolorientierte Zugänge zur Schöpfungs- und Exodusgeschichte bieten sich hier ebenso an wie die Geschichten, in denen Menschen durch göttliche Führung geleitet, bewahrt und gerettet werden. Schliesslich kann und sollte die Lebensgeschichte des Gottessohnes Jesus Christus selbst Modell und Grund vertrauensvollen Erzählens werden, insofern die Evangeliumsbotschaft «im Kern die Geschichte der Anerkennung eines Leidenden durch den [ist], dem dieser Leidende bedingungslos vertraut hat, auf den hin er sein Leben entworfen hat und auf den hin die Gemeinde das Leben dieses Leidenden erzählt» ${ }^{6}$.

$\mathrm{Zu}$ achten ist dabei darauf, dass diese Geschichten nicht nur altersgemäss, sondern zugleich auch so erzählt werden, dass eine aktive und kritische Auseinandersetzung möglich wird. Dabei wird im Einzelfall sicherlich nicht eindeutig und einlinig von vorneherein festzulegen sein, was Kinder und Jugendliche etwa unter einem Gott, dem man vertrauen kann, verstehen. Wichtig ist aber in jedem Fall, diese vor allen Angst machenden oder sie einengenden Gottesbildern zu schützen und ihnen im Gegenteil selbst die Hoffnung auf den annehmenden und versöhnenden Gott vor Augen zu führen. Zur Vertrauens-Bildung gehört dabei zugleich, Kinder und Jugendliche erfahren zu lassen, dass sie sich eigenständig und kritisch auf der Grundlage ihrer eigenen Fragen unterschiedlichen Überlieferungstraditionen und Gottesbildern annähern

6 L. Kuld, Lebensgeschichte(n) - Glaubensgeschichte(n), in: G. Bitter u.a. (Hg.), Neues Handbuch religionspädagogischer Grundbegriffe, München 2002, 178 f. 
dürfen. Biblische Texte und Personen machen ein «tief gefächertes Identifikationsangebot, das von der Rebellion gegen Gott bis hin zum tiefen Vertrauen reicht» ${ }^{7}$.

Die Thematisierung der Gottesfrage muss somit selbst einen prozessual-ergebnisoffenen Charakter tragen. Dies heisst dann auch, dass alle Formen eines verkündigungsorientierten Unterrichts ihrerseits nur dann legitim sein können, wenn sie von Beginn an dialogischen Charakter tragen und eine gemeinsame Auseinandersetzung der Lehrenden und Lernenden mit den unterschiedlichen Überlieferungen befördern. Zudem kann der Theologie und den Theologen nichts Besseres passieren, als wenn ihre eigenen Hypothesen immer wieder auf den Prüfstand gestellt werden, selbst wenn es sich bei den Prüfenden auf den ersten Blick um keine argumentationsfesten Theologen handelt.

Eine solche Vertrauens-Bildung beinhaltet damit konsequenterweise, dass der Lehrende in authentischer Weise seine eigenen Vorstellungen und auch Schwierigkeiten mit bestimmen Geschichten, Bildern und Interpretationen mit einfliessen lässt und zugleich zum Ausdruck bringt, in welcher Weise er sich selbst von der Gewissheit seines eigenen christlichen Glaubens getragen fühlt und weiss. Vertrauens-Bildung kann somit im Blick auf die Gottesfrage produktive und im guten Sinn riskante Kreativität entbinden. In diesem Sinn hat auch die zukünftige Plausibilität der Institution Kirche durchaus mit der Glaubwürdigkeit ihrer RepräsentantInnen zu tun und übersteigt damit die Dimension personaler Vertrauens-Bildung notwendigerweise.

\section{Lässt sich Vertrauens-Bildung evaluieren? - Zur Frage pädagogischer Nachhaltigkeit}

Eine der brennenden Herausforderungen für gegenwärtige religiöse Bildung besteht darin, die spezifische Bedeutsamkeit und Nachhaltigkeit möglichst plausibel transparent zu machen. Dies hängt nicht zuletzt damit zusammen, dass sich angesichts knapper werdender Finanzen im Bildungssektor auch dieser Bereich legitimieren muss. Nun machen die vorherigen Ausführungen deutlich, dass sich bestimmte Prozesse und Dynamiken religiöser Bildung nicht einfach im Modus der Quantifizierung evaluieren lassen. Zwar lassen sich bestimmte Kenntnisse und auch Kompetenzen im

7 H. Aldebert, Anspiel - Rollenspiel - Bibliodrama, in: G. Bitter u.a. (Hg.), Neues Handbuch religionspädagogischer Grundbegriffe, München 2002, 506. 
Umgang mit religiösen Themen und Sachverhalten abprüfen, aber die eigentliche Substanz einer Vertrauensbildung und seine affektive Dimension entziehen sich der Sache nach einer zweifelsfreien Evaluation ${ }^{8}$.

Denkbar und machbar ist allerdings der Versuch, Kinder und Jugendliche ihre eigenen Erfahrungen etwa in Form qualitativer Interviews beschreiben zu lassen und diese dann seinerseits über methodisch gesicherte Verfahren zu interpretieren. Insofern stellt die Meinung, wonach religiöse Bildung als «soft education» wissenschaftlich kein ernsthaftes wissenschaftliches Studienobjekt sein könne, kaum mehr als ein lieb gewonnenes Vorurteil dar. Gerade deshalb sind an die Evaluation religiöser Vertrauens-Bildung die gleichen Massstäbe anzulegen wie an jedes andere Schulfach auch.

Allerdings entstehen hier unter Umständen sehr schnell kognitive Dissonanzen, wenn Erwachsene die Aussagen der Kinder und Jugendlichen nach dem Massstab ihrer eigenen Vorstellungen von gelingender oder gar erfolgreicher Vertrauens-Bildung zu bewerten versuchen.

Zur Freiheit individueller Vertrauens-Bildung gehört somit konsequent auch die Unverfügbarkeit hinsichtlich letztgültiger Beurteilungen dessen, was in Bildungsprozessen geschieht oder eben auch nicht geschieht. Insofern gehört zu einer guten Evaluation religiöser Bildung auch, auf Prozesse der Nachhaltigkeit zu vertrauen, die sich einer zeitnahen Festsetzung entziehen:

«Der Neuorientierung an den Verheißungen steht die Funktionalität einer qualifikations- und kompetenzorientierten Lebenswelt und Erziehung entgegen. Das Vertrauen auf deren Leistungsfähigkeit ist trotz aller Krisen ungebrochen, die Verheißungen des Reiches Gottes erscheinen demgegenüber schwach und vage»".

Gerade deshalb ist aus guten theologischen Gründen allen Versuchen, die Qualität konkreter Bildungsvorgänge vermeintlich eindeutig und abschliessend zu quantifizieren, deutlich und eindeutig $\mathrm{zu}$ widerstehen.

\footnotetext{
${ }^{8}$ Vgl. etwa M. Rothgangel, Religiöse Kompetenz und der Wahrheitsanspruch christlichen Glaubens, in: V. Elsenbast/D. Fischer (Hg.), Stellungnahmen und Kommentare zu «Grundlegende Kompetenzen religiöser Bildung», Münster 2007, 78-81. 9 H. Schmidt, Vertrauen und Verlernen. Glaubensdidaktik angesichts der Krise der Moderne, in: EvTh 50 (1990), 100.
} 


\section{Transdisziplinäre Perspektiven}

Eine religionspädagogische Theoriebildung und Praxisreflexion zur Bedeutung des Vertrauensbegriffs ist unbedingt auf transdisziplinäre Perspektiven angewiesen. Denn nach wie vor scheint die konkrete Praxis in dieser Hinsicht auf einer Reihe von Vorannahmen zu beruhen, die noch keineswegs ausreichend genug ausgelotet sind.

So sind zwar bestimmte entwicklungpsychologische Erkenntnisse - etwa zum Phänomen des Urvertrauens oder der Spannung zwischen Grundvertrauen und Grundmisstrauen (E.H. Erikson) längst in den Problemhorizont der Disziplin eingeflossen. Allerdings werden Erkenntnisse zum Erwerb von Vertrauen etwa aus den Bereichen der Neurobiologie bisher nicht rezipiert oder diskutiert. Insofern zeigt die religionspädagogische Theoriebildung bisher deutlich zu wenig Aufmerksamkeit auf die Bearbeitung anthropologischer Grundeinsichten zur Vertrauensbildung ebenso wie zur Kommunikation und Interaktion von Vertrauensakten, wie dies in anderen Wissenschaftskontexten bearbeitet wird.

Auf der anderen Seite kann die religionspädagogische Bildungstheorie und Bildungsforschung durchaus auch selbst transdisziplinär bereichernd sein, insofern sie über ein erhebliches Mass an praxisorientierter Erfahrung und entsprechender Praxisreflexion in Fragen konkreter Bildungs- und Unterrichtsgestaltung verfügt. Andere Disziplinen können von einer evangelischen Bildungstheorie zum einen wesentliche Erkenntnisse über den Zusammenhang von Theoriebildung und praktisch-didaktischer Implementierung, von Analyse und konkreten didaktischen Handlungsoptionen gewinnen - gemäss der Dilthey'schen Einsicht: «Das letzte Wort des Philosophen ... ist die Pädagogie, denn alles Spekulieren ist um des Handelns willen ${ }^{10}$ - oder derselbe an anderer Stelle: «Das Praktischwerdenkönnen ist ja nun allerdings der eigentliche Rechtsgrund aller Wissenschaft ... Die praktische Abzweckung unseres Standpunktes ist die pädagogische, im weitesten und tiefsten Wortsinne.» ${ }^{11}$

In theologisch-hermeneutischer Hinsicht können damit die Einsichten zur Unverfügbarkeit und gleichzeitigen Verheissung von Bildungsprozessen - und damit auch zur unbedingten Freiheit

${ }^{10}$ W. Dilthey, Pädagogik. Geschichte und Grundlinien des Systems, Ges. Schriften IX, hg. v. O.F. Bollnow, Göttingen ${ }^{5} 2008,203$ f.

${ }^{11}$ Briefwechsel Wilhelm Dilthey und dem Grafen Paul Yorck von Wartenburg 18771897, Halle 1923, 42f. 
individueller Vertrauens-Bildung als Glaubens-Bildung - wichtige Massstäbe für eine öffentliche Bildungs-Kultur liefern. Dies gilt nicht zuletzt dann, wenn Menschen in eine bestimmte von aussen vorbestimmte Richtung so orientiert werden, dass ihre eigene Freiheit dabei nicht mehr zur Geltung kommen kann, sondern alles Handeln in antizivilisatorischem Sinn unter Massgabe prinzipiellen Misstrauens geschieht.

In diesem Sinn liefert auch die anfangs erwähnte Missbrauchsdebatte ein wesentliches Warnsignal hinsichtlich aller Versuche, Menschen durch eine bestimmte selbst gesetzte Autorität für bestimmte Interessen zu funktionalisieren und $\mathrm{zu}$ entmündigen, indem ihnen die Möglichkeit der eigenen freien Wahrheitssuche entzogen und die individuelle Freiheit genommen wird, sich eigenständig denjenigen Orientierungskräften anzuvertrauen, denen sie sich anzuvertrauen bereit sind. Oder wie es der Odenwaldschul-Erfahrene Pädagoge O.F. Bollnow formulierte:

«Dieser Mut zum Vertrauen, dieser Verzicht auf die Sicherung des eigenen Lebens ist notwendig, denn allein in ihm geht der Mensch aus sich heraus und kommt zur Berührung mit einem Leben außer ihm, und erst in dieser Berührung kann sich dann Gemeinschaft und überhaupt eine geistige Welt aufbauen» ${ }^{12}$.

Eine Vertrauens-Bildung im Zielhorizont eines neuen Seinsvertrauens und grösstmöglicher wechselseitiger Anerkennung - durchaus im Licht von Mt 18,3 - bleibt riskant, sie kann und sollte allerdings um gelingender Beziehungen willen ebenso wie aufgrund der Hoffnung als Vertrauen auf eine gute Zukunft gewagt werden.

- Dr. Thomas Schlag ist Assistenzprofessor für Praktische Theologie mit den Schwerpunkten Religionspädagogik und Kybernetik an der Universität Zürich.

\footnotetext{
12 O.F. Bollnow, zit. nach W. Loch, Die Macht des Vertrauens. Otto Friedrich Bollnows anthropologische Pädagogik, in: F. Kümmel (Hg.), O.F. Bollnow. Hermeneutische Philosophie und Pädagogik, Freiburg/München 1997, 222, vgl. auch J. Schwartländer (Hg.) in Verbindung mit M. Landmann u. W. Loch, Verstehen und Vertrauen. Otto Friedrich Bollnow zum 65. Geburtstag, Stuttgart u.a. 1968.
} 